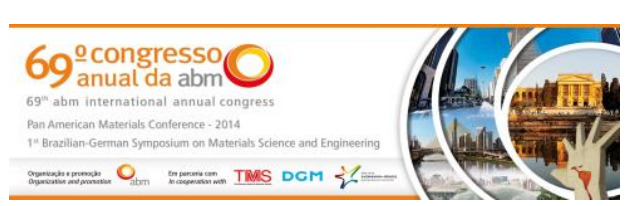

Tema: Gestão de meio ambiente e recuperação e tratamento de rejeitos

\title{
ESTUDO DE ROTA HIDROMETALURGICA VISANDO A EXTRAÇÃO DE PRATA ESTANHO DE SOLDA PRESENTE EM PCl*
}

\section{Resumo}

\author{
Marcos Paulo Kohler Caldas ${ }^{1}$ \\ Ana Paula de Matos Silva² \\ Viviane Tavares de Moraes ${ }^{3}$ \\ Denise Crocce Romano Espinosa 4 \\ Jorge Alberto Soares Tenório ${ }^{4}$
}

Após a edição da RoHS Directive 2002/95/EC, diretiva que entrou em vigor em 1ำ de julho 2006 proibindo a utilização de seis substâncias perigosas: chumbo, mercúrio, cádmio, cromo hexavalente, bifenilos polibromados e éteres difenil-polibromados, fabricantes de equipamentos eletroeletrônicos passaram a utilizar soldas livres de chumbo (lead free) para montagem de suas placas de circuito impresso. A SAC 305 é um tipo de solda muito utilizada pelas empresas fabricantes de placas de circuito impresso. O presente trabalho visa caracterizar este tipo de solda verificando sua composição e identificar se a mesma se encontra livre de elementos perigosos ao ambiente. Avaliou-se a extração de metais como Ag e Sn através de digestão ácida em água-régia e rota $\mathrm{HNO}_{3}$ a temperatura ambiente. A composição do licor lixiviado foi caracterizada por espectrometria de emissão óptica com acoplamento de plasma induzido (ICP-OES). Os resultados indicam que a solda SAC 305 atende a RoHS, possui $2,7 \% \mathrm{Ag}, 96,4 \% \mathrm{Sn}$ e $0,9 \%$ de outros metais e a melhor rota para extração de $\mathrm{Ag}$ foi a $\mathrm{HNO}_{3}$ e para $\mathrm{Sn}$ foi a água-régia.

Palavras-chave: Hidrometalurgia; Reciclagem; Lixiviação; Solda.

\section{STUDY OF HYDROMETALLURGICAL ROUTE AIMING SILVER AND TIN EXTRACTION FROM PCB SOLDER}

\begin{abstract}
In order to meet the RoHS Directive 2002/95/EC (directive which came into force on first of July, 2006 prohibiting the use of these six hazardous substances: lead, mercury, cadmium, hexavalent chromium, polybrominated biphenyls and polybrominated diphenyl ethers) the electronics equipments manufacturers began to use a lead free solder to assembling the Printed Circuit Boards (PCBs). Nowadays, the SAC 305 is a lead free solder type widely used by PCBs manufacturers. So, this paper aims to characterize this solder type to check its composition and identify if it is really free of dangerous components. Were evaluated the $\mathrm{Ag}$ and $\mathrm{Sn}$ extraction by acid digestion in aqua regia and in nitric acid solutions at room temperature $\left(25^{\circ} \mathrm{C}\right)$. The leached liquor composition was determined by optical emission spectrometry with induced coupled plasma (ICP OES). The results indicate that the solder SAC 305 meets the RoHS directive; It have in its composition: $2.7 \%$ w.t. of $\mathrm{Ag}, 96.4 \%$ w.t. of $\mathrm{Sn}$ and $0.9 \%$ w.t. of other metals; and The best route for Ag extraction was nitric acid and for $\mathrm{Sn}$ was aqua regia digestion

Keywords: Hydrometallurgy; Recycling; Leaching; Solder.

\footnotetext{
Doutorando em Engenharia Química, Escola Politécnica, USP, São Paulo, SP, Brasil.

Graduanda, Tecnologia Ambiental, SENAI Mario Amato, São Bernardo do Campo, SP, Brasil.

Doutora em Eng. Metalúrgica e de Materiais, Escola Politécnica, USP, São Paulo, SP, Brasil.
}

Professor(a), Dept. Eng. Química, Escola Politécnica, USP, São Paulo, SP, Brasil.
\end{abstract}

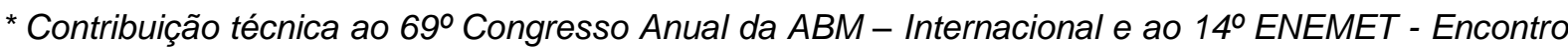
Nacional de Estudantes de Engenharia Metalúrgica, de Materiais e de Minas, 21 a 25 de julho de 2014, São Paulo, SP, Brasil.
} 


\section{INTRODUÇÃO}

A cada ano que passa os equipamentos eletroeletrônicos têm se desenvolvido mais rapidamente com uma redução média de sua expectativa de vida devido, principalmente, às alterações nas suas funções e designs. Esta tendência tem gerado uma grande quantidade de resíduos e, dependendo de sua destinação, torna-se um grave problema ambiental. $\mathrm{Na}$ União Europeia, diretivas foram estabelecidas e tem normatizado o tratamento eficiente dos resíduos de equipamentos eletroeletrônicos [1,2].

Os fabricantes de equipamentos eletroeletrônicos, para atender a norma Europeia de 01 de julho de 2006, estão eliminando elementos perigosos de sua composição. A solda, responsável pelas conexões mecânicas e elétricas de componentes nas placas de circuito impresso ( $\mathrm{PCl}$ ) dos equipamentos, deve ser composta por materiais que ofereçam confiabilidade ao ponto de ligação entre as partes. A liga Sn$\mathrm{Pb}$ foi, durante muitos anos, o sistema mais utilizado na indústria como material de interligação, principalmente devido a confiabilidade da solda e ao seu baixo custo [3]. Porém, devido a toxicidade do chumbo e seu potencial de contaminação de águas subterrâneas, a solda (liga $\mathrm{Sn}-\mathrm{Pb}$ ) utilizada em PCl's, passou a ser substituída por outra composição livre de chumbo (lead free).

As ligas de solda lead free mais utilizadas pela indústria constituem-se principalmente de $\mathrm{Sn}$ com pequenas adições de prata $(\mathrm{Ag})$, cobre $(\mathrm{Cu})$, antimônio (Sb), bismuto (Bi) e zinco (Zi) [4,5]. O chumbo, até então presente na solda $\mathrm{Sn}-\mathrm{Pb}$, passou a ser substituído pela solda $\mathrm{SnAgCu}$, que além de ser livre do chumbo, apresenta temperatura de fusão relativamente baixa comparada com a $\mathrm{Sn}-\mathrm{Pb}$, melhor soldabilidade e características mecânicas [6-8].

As composições mais comuns de solda lead free são a SAC 305 (96,5\% Sn/ 3,0\% $\mathrm{Ag} / 0,5 \% \mathrm{Cu})$, SAC $300(97,0 \% \mathrm{Sn} / 3,0 \% \mathrm{Ag})$, SAC $405(95,5 \% \mathrm{Sn} / 4,0 \% \mathrm{Ag} / 0,5 \%$ Cu) e a SAC $400(96,0 \% \mathrm{Sn} / 4,0 \% \mathrm{Ag})$. As mais apropriadas para substituir a solda $\mathrm{Sn}$-Pb são a SAC 305 e a SAC 405, principalmente devido seu desempenho em condições de fadiga [9].

A quantidade de prata presenta na $\mathrm{PCl}$ é de $0,1 \%$ em massa, mas representa $4,6 \%$ do valor intrínseco da placa [10] e sua concentração nas placas pode chegar a ser até 10 vezes maior que em minerais ricos [11]. Assim a recuperação desse metal torna-se um grande negócio tanto do ponto de vista de mercado como de sustentabilidade.

Utilizando a técnica de lixiviação é possível a transferência de metais presentes em resíduos de equipamentos eletroeletrônicos para o licor através de agentes lixiviantes como soluções acidas, alcalinas e salinas [12].

O objetivo deste trabalho foi realizar a caracterização da solda lead free SAC 305, identificando possíveis rotas para recuperação de $\mathrm{Ag}$ e $\mathrm{Sn}$ e verificar se ela é livre de elementos perigosos a saúde humana e ao meio ambiente.

\section{MATERIAIS E MÉTODOS}

Para o desenvolvimento do trabalho foi utilizada a solda SAC 305, fio cheio de $1,0 \mathrm{~mm}$ doada pelo fabricante.

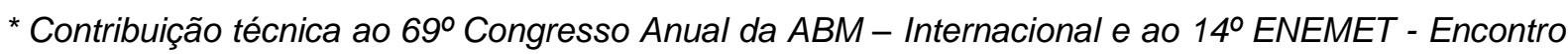
Nacional de Estudantes de Engenharia Metalúrgica, de Materiais e de Minas, 21 a 25 de julho de 2014, São Paulo, SP, Brasil.
} 


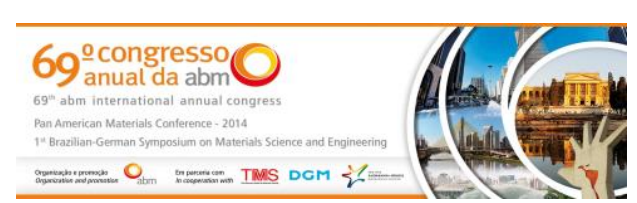

\subsection{Limpeza e Redução do Tamanho}

O verniz presente no fio de solda foi totalmente retirado com o uso de acetona. A amostra de solda foi cortada em pedaços de $1,0 \mathrm{~cm}$ de comprimento. Após a etapa de corte foram separadas duas amostras de $8,0 \mathrm{~g}$ cada que seguiram para os ensaios de digestão.

\subsection{Caracterização Inicial do Fio de Solda}

Com o intuito de caracterizar o fio de solda SAC 305, foram obtidas imagens de microscópio eletrônico de varredura (MEV) e espectroscopia de energia dispersiva (EDS) para quantificar os elementos presentes na amostra.

\subsection{Digestão em Água Régia}

A primeira amostra de $8 \mathrm{~g}$ foi colocada em contato direto com uma solução preparada de água régia (relação de 3:1 para $\mathrm{HCl} \mathrm{HNO}_{3}$ ) na proporção de 1:20 [13] (1 $\mathrm{g}$ de solda para $20 \mathrm{ml}$ de solução de água régia), portanto $8 \mathrm{~g}$ de solda foi colocado em contato direto com $160 \mathrm{ml}$ de solução ácida.

A digestão em água régia foi feita a temperatura ambiente durante 24 horas.

Após esse período o solido não lixiviado foi filtrado do licor lixiviado por meio de filtração simples utilizando-se papel de filtro quantitativo de porosidade $0,75 \mu \mathrm{m}$. $\mathrm{O}$ resíduo não lixiviado foi lavado com água deionizada e mantido em estufa a $60^{\circ} \mathrm{C}+/$ $5^{\circ} \mathrm{C}$ por 24 horas para secar. Após secar foi feita análise de difração de raio x para identificar a composição do sólido não lixiviado.

Uma alíquota de $10 \mathrm{ml}$ foi coletada do licor lixiviado para análise química em ICPOES e determinação da quantidade de metais.

\subsection{Digestão em $\mathrm{HNO}_{3} 10 \mathrm{M}$}

A segunda amostra de $8 \mathrm{~g}$ foi colocada em contato direto com uma solução de $\mathrm{HNO}_{3}$ $10 \mathrm{M}$ na proporção de 1:20 (1 g de solda para $20 \mathrm{ml}$ de solução de $\mathrm{HNO}_{3} 10 \mathrm{M}$ ), portanto $8 \mathrm{~g}$ de solda foi colocado em contato direto com $160 \mathrm{ml}$ de solução ácida.

A digestão em $\mathrm{HNO}_{3} 10 \mathrm{M}$ foi feita a temperatura ambiente durante 2 horas.

Após esse período o solido não lixiviado foi filtrado do licor lixiviado por meio de filtração simples utilizando-se papel de filtro quantitativo de porosidade $0,75 \mu \mathrm{m}$.

Uma alíquota de $10 \mathrm{ml}$ foi coletada para análise química em ICP-OES para determinação da quantidade de metais.

\section{RESULTADOS E DISCUSSÃO}

\subsection{Caracterização Inicial do Fio de Solda}

A Figura 1 apresenta uma imagem obtida por microscopia eletrônica de varredura da superfície do fio de solda. Foi obtido também um espectro de EDS (Figura 2) da região apresentada na Figura 1.

\footnotetext{
* Contribuição técnica ao $69^{\circ}$ Congresso Anual da ABM - Internacional e ao 14ํㅡㄹ ENEMET - Encontro Nacional de Estudantes de Engenharia Metalúrgica, de Materiais e de Minas, 21 a 25 de julho de 2014, São Paulo, SP, Brasil.
} 

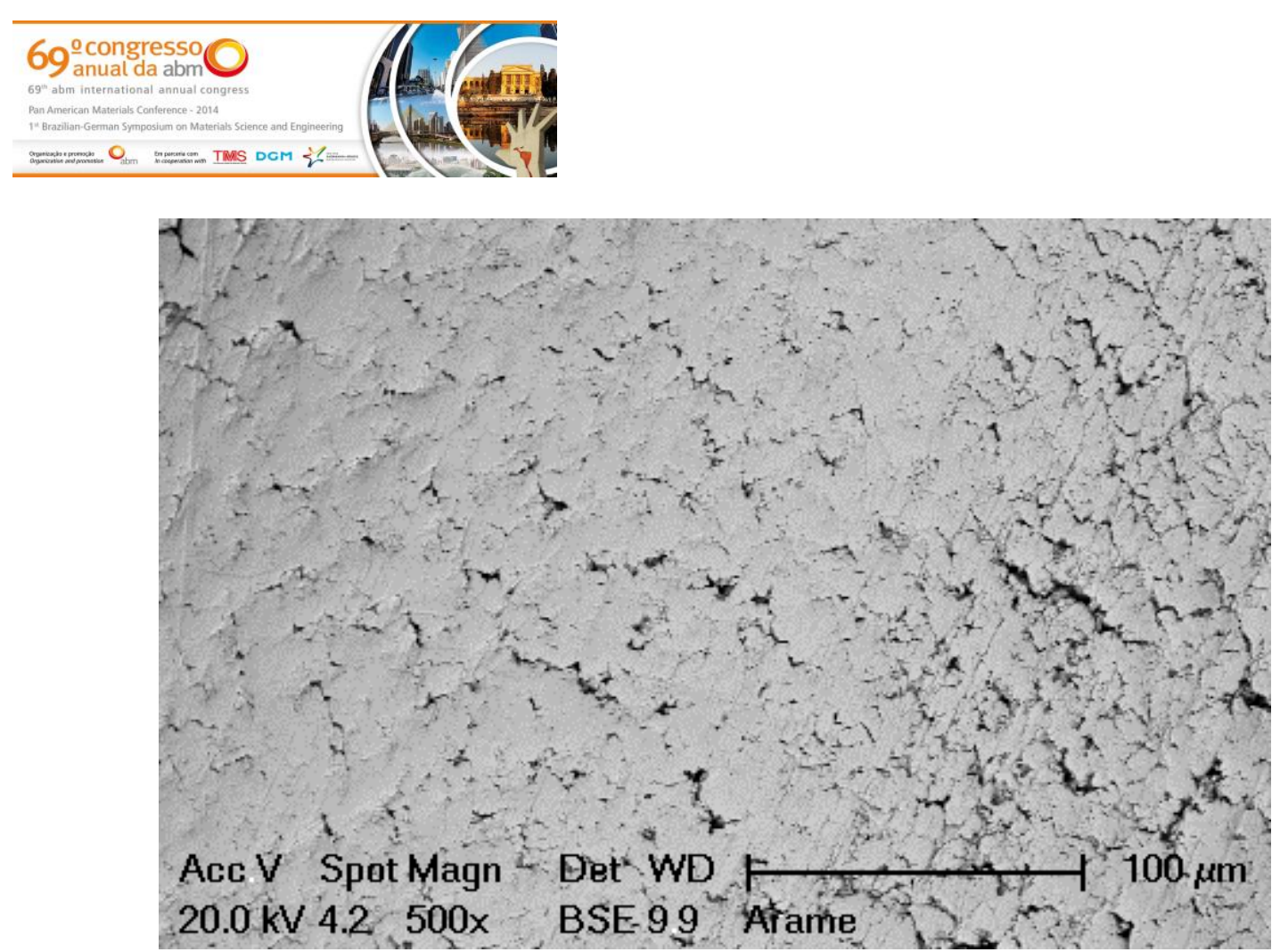

Figura 1. Imagem retro espalhada obtida por microscópio eletrônico de varredura do fio de solda.

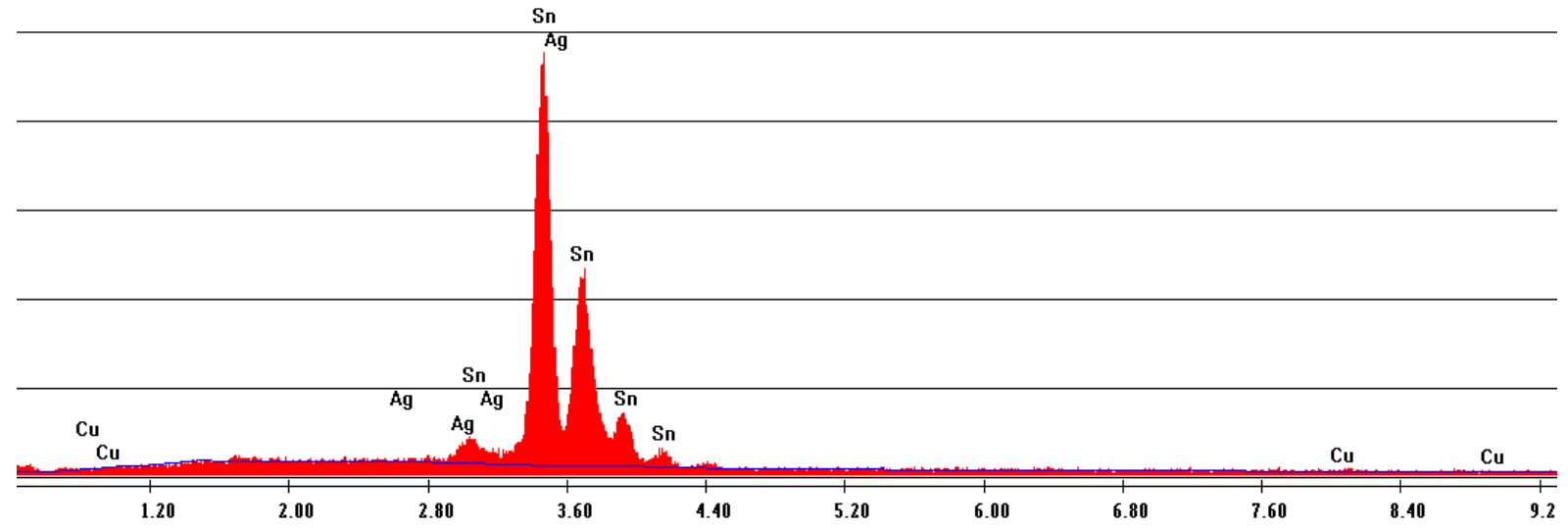

Figura 2. Micrografia eletrônica de varredura do fio de solda SAC 305.

Pelo espectro de EDS pode ser verificado que o fio de solda é composto dos elementos $\mathrm{Sn} \mathrm{Ag}$ e Cu. Foi feito também a quantificação dos elementos identificados no espectro de EDS que podem ser vistos na Tabela 1. A quantificação encontrada é próxima do valor fornecido pelo fabricante $96,5 \% \mathrm{Sn}, 3,0 \% \mathrm{Ag} \mathrm{e} 0,5 \% \mathrm{Cu}$, principalmente para os elementos Sn e Ag que se apresentam em maior quantidade.

Tabela 1. Resultados da quantificação dos elementos identificados no espectro de EDS Metais \% em massa

\begin{tabular}{cc}
\hline $\mathrm{Sn}$ & 96,10 \\
\hline $\mathrm{Ag}$ & 2,75 \\
\hline $\mathrm{Cu}$ & 1,15 \\
\hline
\end{tabular}

\subsection{Digestão em Água Régia}

Os resultados obtidos nas análises de metais (ICP-OES) para a rota de extração por água régia é apresentada na Tabela 2.

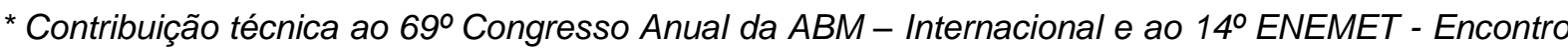
Nacional de Estudantes de Engenharia Metalúrgica, de Materiais e de Minas, 21 a 25 de julho de 2014, São Paulo, SP, Brasil.
} 
Tabela 2. Resultados da análise de metais presente no licor lixiviado da água régia

\begin{tabular}{cc}
\hline Metais & $\begin{array}{c}\text { \% em massa dos } \\
\text { metais em agua } \\
\text { régia }\end{array}$ \\
\hline $\mathrm{Ag}$ & 0,061 \\
\hline $\mathrm{Pb}$ & 0,079 \\
\hline $\mathrm{Sn}$ & 96,373 \\
\hline
\end{tabular}

Geralmente a água régia dissolve a maioria dos metais, porém a prata se apresenta estável $[13,14]$ como pode ser visto no resultado da análise química, Tabela 2 . Na digestão em água régia apenas $2,03 \%$ em massa da prata entrou em solução. Após análise de $\mathrm{DRX}$ do resíduo filtrado da digestão em água régia, Figura 3 , pode-se verificar que o resíduo não lixiviado consiste em $\mathrm{AgCl}$ [13], confirmando o resultado da análise química que também apresentou estabilidade da prata em água régia.

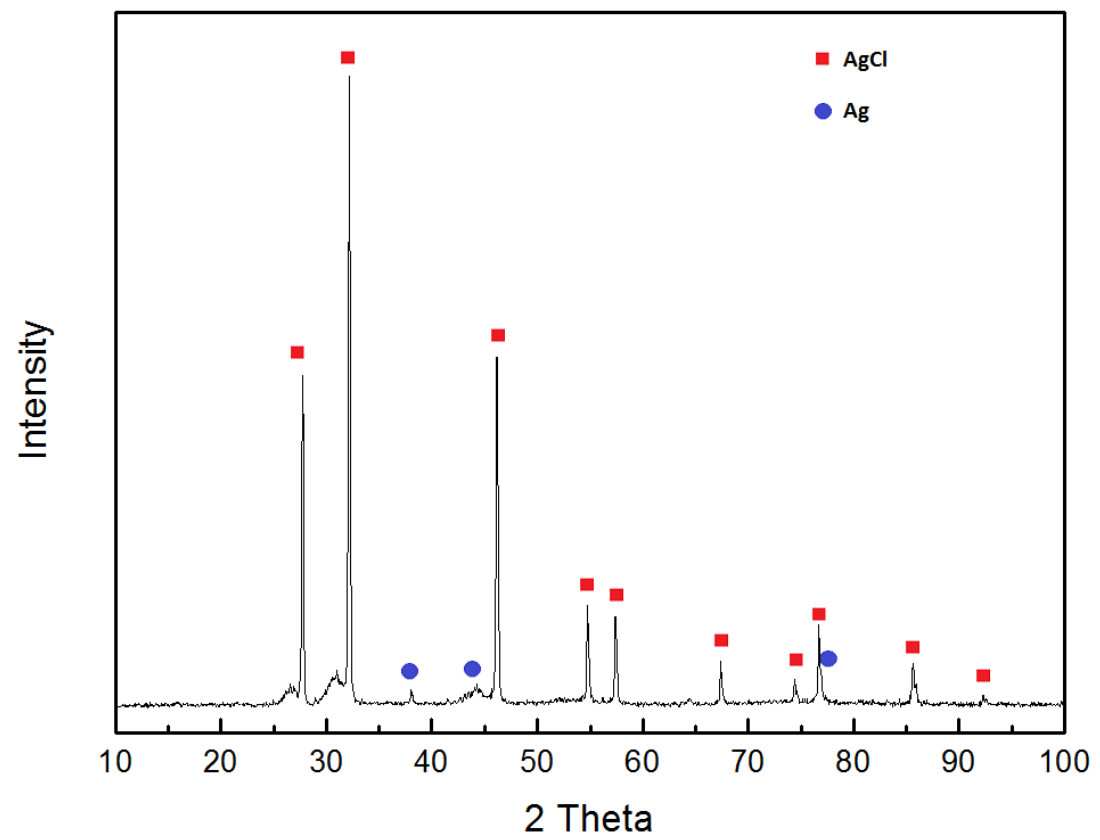

Figura 3. Difratograma de raio $x$ do solido não lixiviado após contato com água régia.

Pela comparação da análise química com a caracterização inicial do fio de solda pode-se verificar que o Sn foi completamente solubilizado em água régia, o que possibilita sua recuperação por esta forma de digestão.

\subsection{Digestão em $\mathrm{HNO}_{3}$ 10M}

Os resultados obtidos nas análises de metais (ICP-OES) para a rota $\mathrm{HNO}_{3}$ de extração são apresentados na Tabela 3.

Tabela 3. Resultados das análises de metais presentes nas amostras de fio de solda para rota HNO3

\begin{tabular}{cc}
\hline Metais & $\begin{array}{c}\text { \% em massa } \\
\text { dos metais em } \\
\mathrm{HNO}_{3}\end{array}$ \\
\hline $\mathrm{Ag}$ & 2,708 \\
\hline $\mathrm{Pb}$ & 0,081 \\
\hline
\end{tabular}

* Contribuição técnica ao $69^{\circ}$ Congresso Anual da ABM - Internacional e ao 14ํㅡㄹ ENEMET - Encontro Nacional de Estudantes de Engenharia Metalúrgica, de Materiais e de Minas, 21 a 25 de julho de 2014, São Paulo, SP, Brasil. 


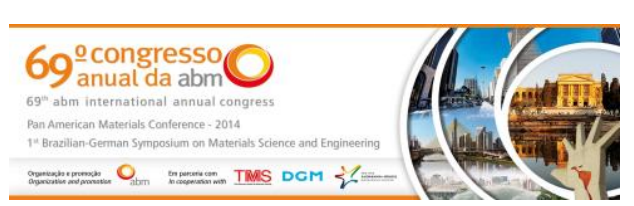

Pela comparação da análise química com a caracterização inicial do fio de solda pode-se verificar que a $\mathrm{Ag}$ foi completamente solubilizada em $\mathrm{HNO}_{3} 10 \mathrm{M}$, o que possibilita sua recuperação por esta forma de digestão. Pela Tabela 3 pode-se verificar também que o Sn não foi solubilizado. Da mesma forma o solido não lixiviado foi filtrado e o Sn surgiu em forma de precipitado de nitrato de estanho.

\section{CONCLUSÃO}

Os resultados obtidos indicam que a solda SAC 305 atende a RoSH, pois conforme análise química (ICP-OES) e quantificação dos elementos identificados no espectro de EDS, a quantidade de substâncias perigosas é inferior ao máximo permitido pela diretiva.

Pode-se concluir também que a melhor rota para recuperação de $\mathrm{Ag}$ é a $\mathrm{HNO}_{3} \mathrm{com}$ $90,33 \%$ de recuperação e para o Sn foi a água régia com 99,89 \% de recuperação.

Considerando as placas de cirucito impressos lead free possuirem principalmente solda SAC 305 os resultados mostram que a caracterização das PCls somente por rota com água regia não é conclusiva para a identificação de prata e que seria necessária uma rota composta de 2 sequencias de digestão uma com água regia e outra com ácido nítrico $10 \mathrm{M}$, pois somente a rota com $\mathrm{HNO}_{3}$ também não foi conclusiva para estanho.

Desta forma, conlui-se que a rota ideal para caracterização de PCls deve serguir a rota de digestão em água régia e $\mathrm{HNO}_{3} 10 \mathrm{~m}$.

\section{Agradecimentos}

Instituto Federal do Espírito Santo - IFES Campus Serra.

Departamento de Engenharia Metalúrgica e de Materiais - Escola Politécnica Universidade de São Paulo.

\section{REFERÊNCIAS}

1 Fauve-Buresi $\mathrm{H}$. Challenging year for the ICT industry for WEEE and RoHS. In: Proceedings of the 5th International Electronics Recycling Congress; 2006; Hamburg, Germany.

2 Europa. Waste Electrical and Electronic Equipment. 2013 [acesso em 26 dez. 2013]. Disponível em: http://ec.europa.eu/environment/waste/weee/index en.htm.

3 Zou C, GaoY, Yang B, Zhai Q. Melting and solidification properties of the nanoparticles of Sn3.0Ag0.5Cu lead-free solder alloy. Materials Characterization. 2010;61:474-480.

4 Arbor A. Lead-free Solder Project Final Report, NCMS Report 0401RE96. National Center for Manufacturing Sciences, 3025 Boarwalk; 1997.

5 Richards BP, Levoguer CL, Hunt CP, Nimmo K, Peters S, Cusack P. Lead free soldering - An analysis of the current status oflead-free soldering. British Department of Trade and Industry Report; 1999.

6 Dong W, Shi Y, Lei Y, Xia Z, Guo F. Effects of small amounts ofNi/ P/Ce element additions on the microstructure and properties of Sn3.0Ag0.5Cu solder alloy. J Mater Sci Mater Electron. 2009;20:1008.

7 Zhang SS, Zhang YJ, Wang HW. Effect of particle size distributions on the rheology of $\mathrm{Sn} / \mathrm{Ag} / \mathrm{Cu}$ lead-free solder pastes. Mater Des. 2010;31:594.

8 Amagai M, Watanabe M, Omiya M, Kishimoto K, Shibuya T. Mechanical characterization of Sn-Ag-based lead-free solders. Microelectron Reliab. 2002;42:951.

\footnotetext{
* Contribuição técnica ao $69^{\circ}$ Congresso Anual da ABM - Internacional e ao 14ํㅡㄹ ENEMET - Encontro Nacional de Estudantes de Engenharia Metalúrgica, de Materiais e de Minas, 21 a 25 de julho de 2014, São Paulo, SP, Brasil.
} 


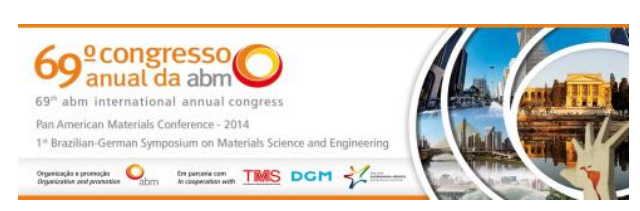

9 Andersson C, Lai Z, Liu J, Jiang H, Yu Y. Comparison of isothermal mechanical fatigue properties of lead-free solder joints and bulk solders. Materials Science and Engineering A. 2005;394:20-27.

10 Goosey M, Kellner R. End-of Life Printed Circuit Boards. 2002. Disponível em: http://www.cfsd.org.uk/seeba/TD/reports/PCB Study.pdf.

$11 \mathrm{Li} \mathrm{J,} \mathrm{Lu} \mathrm{H,} \mathrm{Guo} \mathrm{J,} \mathrm{Xu} \mathrm{Z,} \mathrm{Zhou} \mathrm{Y.} \mathrm{Recycle} \mathrm{Technology} \mathrm{for} \mathrm{Recovering} \mathrm{Resources} \mathrm{and}$ Products from Waste Printed Circuit Boards. Environmental Science Technology. 2007;41:1995-2000.

12 Volsky A, Siergievskaya E. Theory of Metallurgical Processes. Moscow: Mir Publishers; 1978.

13 Park YJ, Fray DJ. Recovery of high purity precious metals from printed circuit boards. Journal of Hazardous Materials. 2009;164:1152-58.

14 Kirk-Othmer Encyclopedia of Chemical Technology. 4th ed. New York: John Wiley \& Sons; 1998.

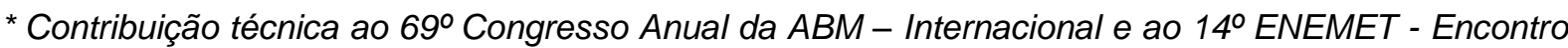
Nacional de Estudantes de Engenharia Metalúrgica, de Materiais e de Minas, 21 a 25 de julho de 2014, São Paulo, SP, Brasil. 